\title{
Effets de la substitution totale du tourteau d'arachide par la fève de coton glandless sur les performances zootechniques de poulets de chair au Sénégal
}

\author{
M.T. Diaw ${ }^{1}$ A. Dieng ${ }^{1}$ G. Mergeai ${ }^{2}$ \\ A. Camara ${ }^{1}$ J.L. Hornick ${ }^{3 *}$
}

Mots-clés

Volaille - Poulet de chair - Coton glandless - Alimentation -

Performance animale - Sénégal.

\begin{abstract}
Résumé
Les performances de poulets de chair recevant un aliment dans lequel le tourteau d'arachide a été totalement substitué par la fève de coton glandless (FCG) ont été comparées à celles d'animaux recevant un aliment témoin expérimental (Tém_E), un aliment de commerce (Tém_C) et une ration de formulation très simple composée uniquement de maïs, de FCG, et de concentré minéral et vitaminé. Dès le démarrage, les animaux Tém_C et FCG ont présenté les meilleures croissances. Après 43 jours d'élevage, les différences de poids vifs ont été très importantes entre les lots, essentiellement suite aux différences de consommation alimentaire, de telle sorte que les meilleures vitesses de croissance n'ont pas été nécessairement liées à de meilleurs indices de consommation. De faibles performances pondérales, de faibles ingestions et des mortalités importantes ont été observées avec la ration simplifiée.
\end{abstract}

\section{INTRODUCTION}

Le cotonnier est la première plante textile au monde et l'espèce Gossypium hirsutum assure 95 p. 100 de la production mondiale de coton. Cette dernière atteint actuellement 27 millions de tonnes (6). L'égrenage du coton graine donne 40 p. 100 de fibre et 60 p. 100 de graines constituées d'amande ( 50 p. 100), de coque (40 à 45 p. 100) et de linter ou fin duvet résiduel recouvrant la graine après arrachage des fibres (8).

Le coton, en tant que matière première oléoprotéagineuse, est peu connu. Pourtant, bien que la trituration des graines et le raffinage de l'huile brute soient des opérations délicates, l'huile de coton,

\footnotetext{
1. Université de Thiès, Ecole nationale supérieure d'agriculture, département Productions animales, Thiès, Sénégal.

2. Université de Liège, Agro-Bio Tech, département Phytotechnie tropicale et horticulture, Gembloux, Belgique.

3. Université de Liège, Faculté de médecine vétérinaire, département Productions animales, 20 Boulevard de Colonster, B 43, 4000 Liège, Belgique.

* Auteur pour la correspondance

Tél. : +32 (0)43664131; fax : +32(0)43664122

E-mail : jlhornick@ulg.ac be
}

avec 4,94 millions de tonnes de production, est au cinquième rang mondial de la consommation d'huile alimentaire devant l'huile de palmiste (4 p. 100) et l'huile d'arachide (3 p. 100). Le tourteau résiduel constitue une source importante de protéine qui le place au deuxième rang mondial derrière le soja. Sa teneur moyenne en protéines est comprise entre 22,2 et 30,3 p. 100 pour le tourteau non décortiqué, et entre 29,7 et 56,0 p. 100 pour le tourteau décortiqué (8).

Cependant, son incorporation dans les rations pour monogastriques, en particulier les volatiles, est limitée par la présence d'un composé polyphénolique, le gossypol qui, sous sa forme libre (GL), diminue la qualité des protéines (2) par sa teneur en fibre (34) et par la présence d'acides gras cyclopropéniques (acides malvalique et sterculique) qui peuvent constituer de puissants inhibiteurs de l'activité enzymatique, comme la delta-9 désaturase intervenant dans la transformation des acides gras (27). De plus, utilisant la fève de coton en remplacement du tourteau d'arachide, Diaw et coll. (6) observent une réduction des performances pondérales et de la digestibilité des nutriments de façon proportionnelle au niveau de substitution. Autrement dit, seul le gossypol contenu dans la fève constituerait un facteur limitant pour son incorporation dans des aliments pour volaille. 
Plusieurs travaux ont été menés pour détoxifier le GL et améliorer la valeur nutritive des rations chez la volaille. Ils se basent essentiellement sur l'apport de fer $(23,24)$ ou de lysine $(2,9,11,31)$, sur l'extraction de l'huile par solvant lors de la trituration (8), sur la supplémentation en acides aminés (31), et sur le développement de variétés de cotonniers exemptes de glandes à gossypol ou variétés glandless $(8,32,33)$.

Cependant, la vulgarisation de tels cotons a connu des difficultés (32) liées à la protection phytosanitaire des cultures dans la mesure où ces glandes protègent la plante contre les ravageurs. Une solution à ce problème serait la création de variétés de cotonniers qui présentent des glandes à gossypol sur toute leur partie aérienne, excepté la graine. Aujourd'hui, les gènes responsables d'un tel caractère ont été identifiés pour leur intégration dans les variétés commerciales (18).

C'est dans cette optique que des travaux de substitution partielle (jusqu'à 75 p. 100) du tourteau d'arachide par la fève de coton glandless ont été effectués dans le passé (7). Les résultats obtenus ont montré une amélioration du poids des animaux proportionnelle au niveau d'incorporation de la fève de coton, laissant espérer des performances encore meilleures en cas de substitution totale. La présente étude a eu ainsi pour objectifs de déterminer les performances pondérales qui permettraient une substitution totale du tourteau d'arachide (couramment utilisé dans les provendes au Sénégal) par la fève de coton glandless, et d'évaluer les effets induits par une ration simplifiée qui serait davantage à la portée des éleveurs amateurs.

\section{MATERIEL ET METHODES}

\section{Production de la fève de coton}

Une production de coton a été entreprise à partir de graines de variétés exemptes de glandes à gossypol (GL7) dans la station expérimentale de l'Ecole nationale supérieure d'agriculture (Ensa) de Thiès (Sénégal). Le coton graine produit a été égrené par la Société de développement des fibres textiles (Sodefitex) du Sénégal. Le tamisage du produit obtenu, après passage au moulin muni d'un tamis de maille $0,7 \mathrm{~cm}$, a donné de la fève de coton dont la valeur nutritionnelle a été rapportée par Diaw et coll. (7).

\section{Conduite des animaux}

Les travaux ont été menés au Centre d'application des techniques d'élevage de l'Ensa. Le climat y est de type soudano-sahélien avec une alternance de saisons pluvieuse (juillet à septembre/octobre) et sèche (novembre à juin).

Durant l'essai, qui a coïncidé avec la saison pluvieuse, la température et l'humidité ont été quotidiennement déterminées dans le poulailler avec des mesures le matin $(7 \mathrm{~h} 00)$ et à la mi-journée (13 h 00). Une température moyenne de $31{ }^{\circ} \mathrm{C}$ a été enregistrée avec respectivement un minimum de $19{ }^{\circ} \mathrm{C}$ et un maximum de $35^{\circ} \mathrm{C}$. L'humidité relative moyenne correspondante de l'air a été de 70 p. 100 avec un maximum de 89 p. 100 et un minimum de 45 p. 100.

L'essai a porté sur 200 poulets d'un jour, non-sexés et de souche Cobb 500, réputée à croissance rapide (29). Les poussins ont été répartis, dès leur réception, en quatre lots de 50 animaux, divisés chacun en deux répétitions. Un lot témoin (témoin expérimental, Tém_E) a reçu un aliment comportant 25 p. 100 de tourteau d'arachide. Un deuxième lot a été nourri avec le même aliment que le Tém_E mais le tourteau d'arachide a été totalement remplacé par la fève de coton glandless (FCG). Un troisième lot a reçu un aliment de formule très simple pouvant être vulgarisée auprès des petits éleveurs (aliment simplifié, A_simplif). Enfin, un quatrième lot a reçu un aliment de référence homologué de commerce (témoin commercial, Tém_C).

Une formule démarrage a été distribuée au niveau de chaque lot $\mathrm{du} 1^{\mathrm{er}}$ au $21^{\mathrm{e}}$ jour. Du $22^{\mathrm{e}}$ jour jusqu'à la fin de l'essai (43 ${ }^{\mathrm{e}}$ jour), les animaux ont reçu un aliment croissance. Les aliments ont été formulés pour présenter des teneurs iso-protéiques et iso-énergétiques. Les niveaux d'extrait éthéré (EE) ont été ajustés à ceux du lot FCG par l'apport d'huile d'arachide (tableau I). Les teneurs en nutriment ont été calculées sur la base des recommandations du National Research Council (20) avec un rapport énergie/protéine moyen de 135 au démarrage et de 159 en croissance, et environ $3100 \mathrm{kcal}$ d'énergie métabolisable (EM) par kilogramme d'aliment. Durant toute la période expérimentale, la distribution d'eau et d'aliments a été faite ad libitum.

Les poids vifs individuels ont été déterminés de façon hebdomadaire jusqu'à la fin de l'essai. Des mesures quotidiennes de la prise alimentaire par lot et des mortalités ont également été effectuées. Le programme prophylactique appliqué durant la période expérimentale a été le même que celui en vigueur au Sénégal dans la zone expérimentale.

\section{Analyses chimiques et calculs}

La détermination des teneurs en matière sèche (MS), cendre $(\mathrm{Ce})$, cellulose brute $(\mathrm{CB})$ et extrait éthéré (EE) a été faite conformément aux méthodes de l'AOAC (1). Les protéines brutes (PB) ont été déterminées par la méthode Kjeldahl. L'énergie métabolisable (EM) a été évaluée par une équation de l'Inra (15) où l'EM vraie $(\mathrm{Mj} / \mathrm{kg} \mathrm{MS})=(3951+54,4 * \mathrm{EE}-88,7 * \mathrm{CB}-40,8 * \mathrm{Ce})$ $* 0,004184$, les teneurs en nutriments étant exprimées en pourcentage de la MS.

\section{Analyse des données}

Pour les poids vifs, les données ont été individuelles et analysées selon la procédure du modèle linaire généralisé (GLM) de SAS (25). Les données ne pouvant être individualisées (gain quotidien, consommation alimentaire, indice de consommation et mortalité) ont fait l'objet d'une simple statistique descriptive. Le modèle utilisé a inclu l'effet du lot, de la répétition et de leur interaction (lot*répétition), et a été le suivant :

$$
\mathrm{Y}=\mu+\alpha \mathrm{i}+\beta \mathrm{j}+\alpha \beta \mathrm{ij}+\varepsilon \mathrm{ijk}
$$

où $\mu$ est la moyenne générale, $\alpha \mathrm{i}$ l'effet du lot $\mathrm{i}, \beta \mathrm{j}$ l'effet de la répétition $\mathrm{j}$, $\alpha \beta \mathrm{ij}$ l'effet de l'interaction lot*répétition, et عijk l'écart résiduel aléatoire.

\section{RESULTATS}

L'évolution du poids vif des animaux dans chaque lot est montrée dans la figure 1 . Le poids moyen à la fin des phases démarrage et croissance a été significativement affecté $(\mathrm{p}<0,001)$ par le type d'aliment distribué (tableau II). L'effet de la répétition n'a pas été significatif $(\mathrm{p}=0,65)$.

L'évolution du poids des animaux du lot Tém_C a été exponentielle, alors que dans les autres lots cette croissance a été sigmoïdale (figure 1). Les animaux Tém_C et FCG ont présenté les meilleures performances dès la fin de la deuxième semaine (J15) où, avec des poids moyens respectifs de 257 et $225 \mathrm{~g}(\mathrm{p}=0,23)$, ils se sont distingués significativement des animaux Tém_E et A_simplif ( $p<0,001)$. Durant toute la phase démarrage, aucune différence 
Tableau I

Composition des aliments utilisés

\begin{tabular}{|c|c|c|c|c|c|c|c|}
\hline & \multicolumn{3}{|c|}{ Démarrage } & \multicolumn{3}{|c|}{ Croissance } & \multirow[b]{2}{*}{ A_simplif ${ }^{4}$} \\
\hline & Tém_C 1 & Tém_E 2 & FCG $^{3}$ & Tém_C & Tém_E & FCG & \\
\hline \multicolumn{8}{|l|}{ Aliments du mélange (\%) } \\
\hline Maïs & - & 40 & 49,2 & - & 52 & 49,5 & 70 \\
\hline Mil & - & 13 & 9 & - & 10 & 8 & 0 \\
\hline Sorgho & - & 4 & 3 & - & 4 & 3 & 0 \\
\hline Tourteau d'arachide & - & 25 & 0 & - & 25 & 0 & 0 \\
\hline Fève de coton & - & 0 & 25 & - & 0 & 25 & 25 \\
\hline Farine de poisson & - & 8 & 7,3 & - & 0 & 8 & 0 \\
\hline Craie & - & 0,05 & 0,09 & - & 0 & 0 & 0 \\
\hline Phosphate tricalcique & - & 0,25 & 0,01 & - & 0,75 & 0,1 & 0 \\
\hline Huile d'arachide & - & 4,5 & 1,2 & - & 2,72 & 1,2 & 0 \\
\hline Lysine de synthèse & - & 0,15 & 0,15 & - & 0,4 & 0,1 & 0 \\
\hline Méthionine de synthèse & - & 0,05 & 0,05 & - & 0,13 & 0,1 & 0 \\
\hline Macro-vetamix $5 \%{ }^{5}$ & - & 5 & 5 & - & 5 & 5 & 5 \\
\hline \multicolumn{8}{|l|}{ Composition prévue } \\
\hline $\mathrm{EM}(\mathrm{kcal} / \mathrm{kg})$ & - & 3147 & 3134 & - & 3095 & 3128 & 3115 \\
\hline Protéine brute (\%) & - & 22,91 & 22,93 & - & 19,70 & 23,19 & 19,36 \\
\hline Lysine (\%) & - & 1,25 & 1,28 & - & 1,19 & 1,48 & 0,84 \\
\hline Méthionine (\%) & - & 0,54 & 0,56 & - & 0,51 & 0,61 & 0,40 \\
\hline Calcium (\%) & - & 1,97 & 1,87 & - & 1,73 & 1,91 & 1,46 \\
\hline Phosphore (\%) & - & 0,48 & 0,73 & - & 0,38 & 0,76 & 0,55 \\
\hline Extrait éthéré (\%) & - & 9,36 & 13,79 & - & 7,68 & 13,79 & 12,68 \\
\hline Cellulose brute (\%) & - & 5,17 & 6,10 & - & 5,43 & 6,08 & 6,39 \\
\hline \multicolumn{8}{|l|}{ Composition analysée } \\
\hline EM $(\mathrm{kcal} / \mathrm{kg})$ & 3107 & 3076 & 3263 & 3135 & 2937 & 3045 & 3002 \\
\hline Protéine brute (\%) & 22,48 & 23,64 & 21,63 & 21,22 & 19,20 & 20,67 & 19,37 \\
\hline Extrait éthéré (\%) & 2,37 & 6,73 & 5,83 & 3,56 & 5,90 & 4,45 & 4,50 \\
\hline Cellulose brute (\%) & 4,55 & 7,67 & 5,53 & 5,00 & 7,90 & 6,49 & 5,58 \\
\hline Rapport EM/PB & 138 & 130 & 150 & 148 & 153 & 147 & 155 \\
\hline
\end{tabular}

${ }^{1}$ Tém_C : témoin commercial $;{ }^{2}$ Tém_E : témoin expérimental contenant $25 \%$ de tourteau d'arachide ; ${ }^{3}$ FCG : aliment où le tourteau d'arachide a été totalement remplacé par la fève de coton glandless ; ${ }^{4}$ A_simplif : aliment simplifié composé uniquement de 3 ingrédients ; ${ }^{5}$ Par kilogramme de Premix : Ca, $250 \mathrm{~g} ; \mathrm{P}, 49 ; \mathrm{NaCl}, 33 ; \mathrm{Mn}, 1,4 \mathrm{~g}$; $\mathrm{Zn}, 1,2 \mathrm{~g} ; \mathrm{Fe}, 1,4 \mathrm{~g}$; Cu 0,2 g; I , 8 ppm ; Co, 2 ppm ; Se, 2,8 ppm ; vitamine A, $250000 \mathrm{UI}$; vitamine D3, $50000 \mathrm{UI}$; vitamine E, $290 \mathrm{mg}$; vitamine B1, $55 \mathrm{mg}$; vitamine $\mathrm{B} 2,100 \mathrm{mg}$; vitamine B3, $480 \mathrm{mg}$; vitamine B5 $195 \mathrm{mg}$; vitamine B6, $55 \mathrm{mg}$; vitamine B12, $600 \mu \mathrm{g}$; vitamine $\mathrm{K} 3,50 \mathrm{mg}$; vitamine acide folique, $27 \mathrm{mg}$; vitamine C, $175 \mathrm{mg}$; H biotine, $600 \mu \mathrm{g}$; lysine $\mathrm{HCl}, 5 \%$; méthionine, $3 \%$; $\mathrm{PB}, 9,2 \%$; MG, $0,9 \%$.

significative $(\mathrm{p}=0,29)$ n'a été observée entre les animaux recevant l'aliment commercial et le lot FCG. Après trois semaines d'essai, leurs poids moyens respectifs ont été de 461 et $489 \mathrm{~g}$ (tableau II).

Les différences observées se sont accentuées en deuxième période mais le lot Tém_C s'est nettement démarqué des autres lots avec, à la fin de l'essai, un poids vif moyen de $1732 \mathrm{~g}$ contre $435 \mathrm{~g}$ pour les animaux A_simplif. Les sujets des lots FCG et Tém_E ont présenté des performances intermédiaires mais différentes $(\mathrm{p}<0,001)$ et ont pesé respectivement 1103 et $916 \mathrm{~g}$.

Le gain moyen quotidien (GMQ) maximal durant la phase démarrage a été enregistré chez les animaux du lot FCG qui a réalisé une croissance de 23,9 g/j, très proche de celle du lot Tém_C $(23,1$ $\mathrm{g} / \mathrm{j})$, tandis que le lot Tém_E a présenté des valeurs intermédiaires $(15,7 \mathrm{~g} / \mathrm{j})$ et la ration simplifiée n'a permis de réaliser que $6,4 \mathrm{~g} / \mathrm{j}$. Pendant la phase de croissance, les meilleurs GMQ $(61,1 \mathrm{~g})$ ont été observés chez les animaux ayant reçu l'aliment commercial.
Comme au démarrage, les poussins du lot A_simplif ont présenté les plus faibles performances $(12,7 \mathrm{~g} / \mathrm{j})$ alors que les lots FCG et Tém_E ont obtenu des valeurs intermédiaires (29,2 et 28,2 g/j).

La prise alimentaire a été plus forte, durant la phase de démarrage, chez les animaux du lot FCG, avec $800 \mathrm{~g}$ par sujet contre 648 et $528 \mathrm{~g}$ respectivement chez les poussins des lots Tém_C et Tém_E. Une faible ingestion a été observée chez les sujets ayant reçu l'aliment simplifié avec $303 \mathrm{~g}$ par animal, soit $15,2 \mathrm{~g} / \mathrm{j}$. Pendant la phase de croissance, l'ingestion a été plus importante chez le Tém_C (2 833 g par sujet ou 142 g/j), dépassant largement celle des volailles des autres lots (tableau II).

L'efficience alimentaire des différentes rations a révélé, pour la phase démarrage, un indice de consommation (IC) plus favorable dans le lot Tém_C $(1,54)$, contre 1,77 et 1,85 , respectivement pour les lots FCG et Tém_E (tableau II). La ration simplifiée a donné un IC de 2,34 pour cette phase. En revanche, cet aliment s'est révélé 
plus efficace durant la phase de croissance où l'efficience a été de 1,89 contre 2,21 pour l'aliment commercial, et de 3,28 et 3,54 respectivement pour les aliments des lots Tém_E et FCG.

De fortes mortalités (tableau II) ont été enregistrées durant l'expérimentation chez les animaux recevant la ration simplifiée ( 24 p. 100 durant le démarrage contre 2 p. 100 pour les animaux Tém_C). Dans ce lot, les pertes se sont maintenues jusqu'à la réduction de l'effectif de presque de moitié (46 p. 100) à la fin de 1'essai (figure 2). Chez les animaux du lot FCG, les pertes enregistrées n'ont pas présenté de différence avec celles des animaux ayant reçu la ration commerciale (2 p. 100 au démarrage, et respectivement 14 et 12 p. 100 durant tout l'essai). Des mortali-

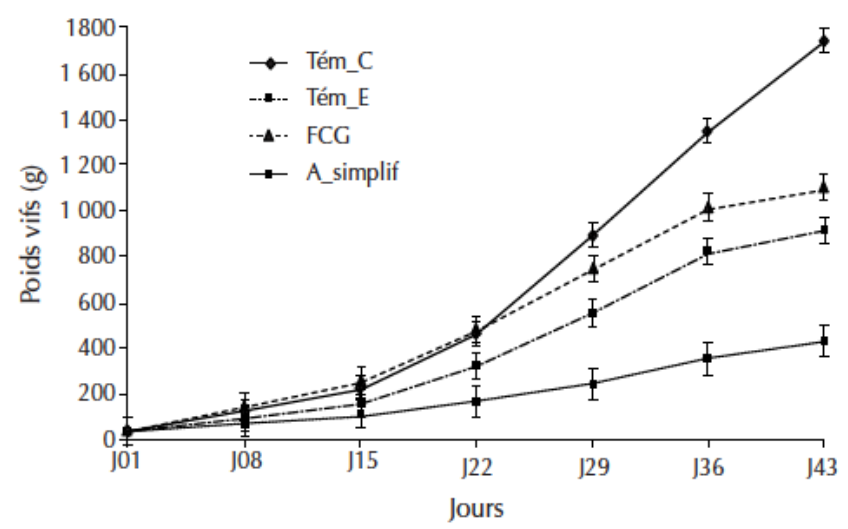

Figure 1 : évolution des performances pondérales de poulets recevant un aliment de type commercial (témoin commercial, Tém_C), ou un aliment témoin contenant 25 p. 100 de tourteau d'arachide (témoin expérimental, Tém_E), ou un aliment où le tourteau d'arachide a été totalement remplacé par la fève de coton glandless (FCG), ou un aliment simplifié à base de maïs et de fève de coton (A_simplif). tés importantes ont été observées chez le témoin expérimental (jusqu'à 26 p. 100 à la fin de l'essai).

\section{DISCUSSION}

Les performances réalisées avec l'aliment commercial ont été meilleures et pourtant, entre les lots, tous les aliments avaient des valeurs nutritionnelles similaires. Il faut signaler que les aliments expérimentaux ont été présentés sous forme de miettes tandis que l'aliment croissance Tém_C a été présenté sous forme de granulés. Or, les meilleures performances ont été notées pendant la croissance, dans le lot Tém_C. L'aspect des aliments pourrait ainsi

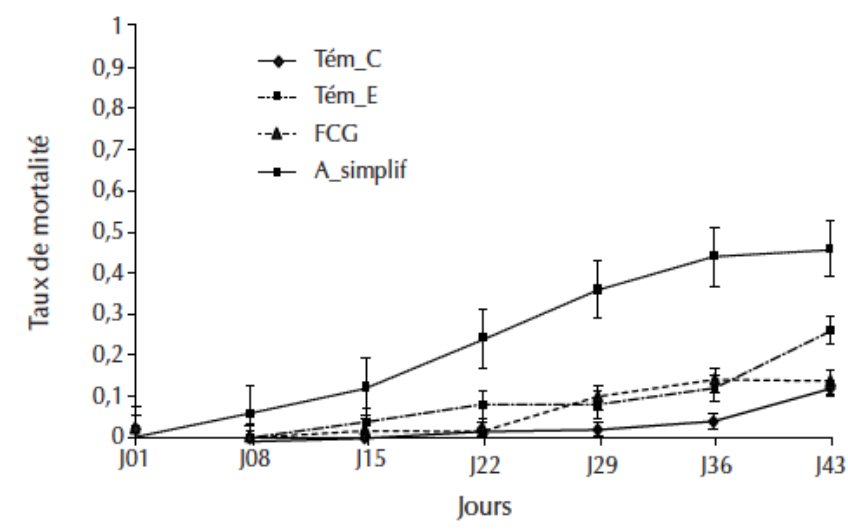

Figure 2 : évolution du taux de mortalité de poulets recevant un aliment de type commercial (témoin commercial, Tém_C), ou un aliment témoin contenant 25 p. 100 de tourteau d'arachide (témoin expérimental, Tém_E), ou un aliment où le tourteau d'arachide a été totalement remplacé par la fève de coton glandless (FCG), ou un aliment simplifié à base de maïs et de fève de coton (A_simplif).

\section{Tableau II}

Performances zootechniques de poulets recevant différents types d'aliments

\begin{tabular}{|c|c|c|c|c|c|c|}
\hline & Tém_C ${ }^{1}$ & Tém_E 2 & FCG $^{3}$ & A_simplif ${ }^{4}$ & ETR $^{5}$ & $P>F$ \\
\hline Poids initial (g) & $38,7^{\mathrm{a}}$ & $39,9^{a}$ & $38,9^{a}$ & $39,8^{a}$ & 2,8 & 0,767 \\
\hline Poids fin démarrage (g) & $460,7^{a}$ & $324,4^{b}$ & $488,8^{a}$ & $168,8^{c}$ & 48,3 & 0,001 \\
\hline Poids final croissance (g) & $1732,2^{a}$ & $916,3^{c}$ & $1102,9^{b}$ & $435,3^{d}$ & 180,1 & 0,001 \\
\hline GMQ démarrage (g/j) & 23,1 & 15,8 & 23,8 & 6,4 & - & - \\
\hline GMQ croissance (g/j) & 61,1 & 28,2 & 29,2 & 12,7 & - & - \\
\hline GMQ cumulé (g/j) & 39,4 & 20,4 & 24,7 & 9,2 & - & - \\
\hline Ingestion totale démarrage (g) & 648 & 527 & 800 & 303 & - & - \\
\hline Ingestion totale croissance (g) & 2833 & 1943 & 2174 & 505 & - & - \\
\hline Ingestion totale $(\mathrm{g} / \mathrm{j})$ & 3481 & 2470 & 2974 & 808 & - & - \\
\hline IC 6 démarrage & 1,53 & 1,85 & 1,77 & 2,34 & - & - \\
\hline IC croissance & 2,21 & 3,28 & 3,54 & 1,89 & - & - \\
\hline IC cumulé & 2,06 & 2,82 & 2,80 & 2,04 & - & - \\
\hline Mortalité démarrage (\%) & 2 & 8 & 2 & 24 & - & - \\
\hline Mortalité croissance (\%) & 10 & 18 & 12 & 22 & - & - \\
\hline Mortalité cumulée (\%) & 12 & 26 & 14 & 46 & - & - \\
\hline
\end{tabular}

Les chiffres avec la même lettre sur une même ligne ne présentent aucune différence significative au seuil de $5 \%$

${ }^{1}$ Tém_C : témoin commercial ; ${ }^{2}$ Tém_E : témoin expérimental contenant $25 \%$ de tourteau d'arachide ; ${ }^{3} \mathrm{FCG}$ : aliment où le tourteau d'arachide a été totalement remplacé par la fêve de coton glandless : ${ }^{4} \mathrm{~A} \_$simplif. : aliment simplifié à base de maïs et de fève de coton, de même formulation en démarrage et en croissance; ${ }^{5}$ ETR : écart-type résiduel ; IC : indice de consommation 
expliquer des différences de consommation (19) qui auraient alors influencé les performances pondérales. Buldgen (3) a toutefois observé que, durant la phase de croissance, la granulation n'avait pas d'effet notable sur les performances des animaux.

De plus, les faibles performances des animaux ayant reçu les aliments expérimentaux pourraient s'expliquer par le fait que les matières premières composant les rations formulées ont été acquises dans le commerce où les conditions de conservation peuvent ne pas être optimales, entraînant l'oxydation éventuelle des lipides ou le développement de mycotoxines dans le tourteau d'arachide. En effet, les mauvaises conditions de conservation du tourteau d'arachide occasionnent le plus souvent le développement d'aflatoxines (B1, B2, G1 et G2) dont la limite de toxicité chez la volaille se situe à $0,25 \mathrm{mg}$ d'aflatoxine $\mathrm{B} 1(\mathrm{AB} 1)$ par kilogramme d'aliment. Czegledi et Gutzwiller (4) rapportent que la limite permise d'AB1 dans les provendes est de 0,030 et $0,050 \mathrm{mg}$ par kilogramme d'aliment volaille, respectivement en Suisse et en France. Etant donné que le dosage des aflatoxines n'a pas été effectué, cette hypothèse ne peut être écartée dans la mesure où d'autres mycotoxines spécifiques au tourteau d'arachide pouvaient également être à l'origine des effets négatifs sur les performances pondérales de ces animaux. Toutefois, les essais menés par Diaw et coll. (7) dans des conditions similaires et ayant entraîné des performances proches n'ont pas mis en évidence des teneurs significatives en aflatoxines dans les provendes de même type.

Plus vraisemblablement, les phénomènes d'oxydation des lipides du mélange pourraient être à l'origine des faibles performances des poulets Tém_E où une quantité importante d'huile a été apportée (4,5 et 2,7 p. 100 respectivement dans les rations démarrage et croissance). En effet, il n'y avait pas d'antioxydant dans les provendes. L'oxydation éventuelle est moins probable avec les fèves de coton car ces dernières sont très riches en vitamine $\mathrm{E}$ qui a un pouvoir antioxydant et les lipides devaient être relativement protégés à l'intérieur des cellules de la fève. Finalement, il n'est pas exclu que l'huile d'arachide ait eu des conséquences négatives sur les animaux. Il faut cependant noter qu'une plus faible digestibilité du tourteau d'arachide, associée à des teneurs en acides aminés inférieures à celles de la fève de coton glandless, pouvait également expliquer les plus faibles performances du témoin expérimental par rapport à l'aliment FCG.

En effet, l'expérimentation a également montré une amélioration du poids vif des animaux lors du remplacement total du tourteau d'arachide par la fève de coton glandless. Des résultats similaires ont été rapportés dans la littérature avec une augmentation proportionnelle des poids des animaux jusqu'à 75 p. 100 de substitution du tourteau d'arachide par la fève de coton glandless (7). En remplaçant le tourteau d'arachide par le tourteau de coton décortiqué et déshuilé au solvant (Tcdds) avec des niveaux de GL très faibles (72 à $289 \mathrm{mg} / \mathrm{kg}$ d'aliment), certains auteurs ont également observé, sur une période de quatre semaines, une amélioration des poids vifs des poulets (26). L'incorporation de Tcdds (400 mg de $\mathrm{GL} / \mathrm{kg}$ d'aliment) à raison de $30 \mathrm{p} .100$ dans les rations pour poulet a donné des performances significativement meilleures que celles avec le tourteau d'arachide (28) comme principal aliment protéique dans la ration démarrage (750 contre $691 \mathrm{~g})$. Toutefois, l'utilisation de la fève de coton glandless comme substitut total du tourteau d'arachide n'a jamais été décrite auparavant, à la connaissance des auteurs, et indique l'excellente valeur alimentaire de cet aliment en production de poulets.

L'extrapolation des résultats de Diaw et coll. (7) sur des essais conduits dans des conditions similaires à la présente étude avait permis d'estimer les performances de poulets recevant une ration où le tourteau d'arachide était substitué totalement par la FCG à $1967 \mathrm{~g}$ après 45 jours d'élevage. Pourtant, les poids vifs enregistrés chez les animaux dans cet essai ont été de $1103 \mathrm{~g}$ après 43 jours d'élevage. En considérant des GMQ de 29 g, le poids à 45 jours atteindrait au maximum $1161 \mathrm{~g}$. Plusieurs hypothèses peuvent être évoquées pour expliquer ces différences. D'une part, par rapport aux essais rapportés par Diaw et coll. (7), la présente période expérimentale a coïncidé avec la saison des pluies où les températures et l'humidité ont été très élevées. D'autre part, de fortes variabilités peuvent exister quant à la qualité des aliments composant les rations. Enfin, il n'est pas impossible qu'un effet favorable linéaire se manifeste jusqu'à des taux de substitution du tourteau d'arachide de l'ordre de 75 p. 100, mais pas au-delà.

Cependant, par rapport aux résultats de Reddy et Eshwaraiah (26), les poids vifs moyens enregistrés après quatre semaines d'élevage ont été satisfaisants chez les animaux où le tourteau d'arachide a été totalement substitué par la fève de coton et chez ceux ayant reçu l'aliment commercial (respectivement 752 et $895 \mathrm{~g}$ contre $618 \mathrm{~g}$ ). A cet âge, certains auteurs (28) ont enregistré des poids similaires avec $750 \mathrm{~g}$ à 30 p. 100 d'incorporation du Tcdds (400 mg de GL/kg d'aliment) en remplacement du tourteau d'arachide. La substitution totale du tourteau de soja par le tourteau de coton issu de divers procédés de fabrication, et donc avec différentes teneurs en GL, donne des poids moyens compris entre 722 et $786 \mathrm{~g}$ respectivement avec des rations de 504 et $92 \mathrm{mg}$ de GL par kilogramme d'aliment (11). Plusieurs auteurs montrent que les performances des animaux dépendent non seulement de la teneur en GL dans la ration mais également des divers traitements de la graine de coton qui influent beaucoup sur la valeur nutritive du tourteau utilisé et donc de l'aliment $(14,19,34)$.

Les GMQ réalisés par les poussins recevant la ration simplifiée ont été très faibles par rapport aux autres lots. Cette ration était constituée de maïs (70 p. 100), de fève de coton (25 p. 100) et de complément minéral vitaminé (Macrovétamix 5 p. 100) pour couvrir les besoins minimums des animaux. Selon Malcolm Reid et coll. (17), une ration optimale pour des poulets renferme une quarantaine de nutriments qui ne peuvent être obtenus qu'en incorporant au moins une dizaine d'aliments dans les mélanges. Cette recommandation n'a pu être respectée dans le cas de la formulation simplifiée. Toutefois, selon les calculs, l'apport d'un complexe minéral - vitaminique, à raison de 5 p. 100 de la ration, a couvert tous les besoins en nutriments des animaux. Le fait que cet aliment n'a permis que des GMQ de l'ordre $10 \mathrm{~g} / \mathrm{j}$ est à attribuer à une très faible appétibilité.

Cette ration a toutefois le mérite d'être facile à vulgariser en milieu rural pour améliorer les conditions d'élevage avicole à l'aide d'un aliment de bonne qualité. Il serait donc intéressant d'étudier les croissances obtenues chez la volaille traditionnelle, caractérisée par un comportement de divagation. Les performances réalisées chez des volailles de ce type recevant un complément protéique et énergétique devraient en effet être significativement améliorées par le fait que les animaux diversifient naturellement leur alimentation à partir de ressources naturelles (5). Plusieurs auteurs $(10,12,30)$ ont rapporté des performances d'environ $1000 \mathrm{~g}$ chez des poulets traditionnels de 10 semaines d'âge. La complémentation alimentaire en divagation est surtout énergétique, et complétée avec des sources protéiques animales telles que les vers de terre et les insectes. La faible productivité observée par la plupart des auteurs est associée à l'absence de l'amélioration génétique de la poule locale $(10,21)$. Cependant, une modification du système de complémentation pourrait aider à rehausser les performances et par conséquent le niveau de revenus générés (12). Ceci pourrait être particulièrement pertinent en zone cotonnière où la formule mériterait d'être étudiée de manière plus approfondie. 
Dans cette expérience, le niveau d'ingestion a été corrélé aux performances de croissance. Ainsi, l'ingestion a été plus élevée chez les animaux recevant l'aliment commercial et la fève de coton glandless en remplacement du tourteau d'arachide. De plus, l'ingestion de la ration FCG a été correcte, en comparaison avec le Tém_C, durant la phase démarrage, ce qui permet de réfuter toute hypothèse d'inappétence de la fève de coton glandless pour expliquer les faibles prises alimentaires des animaux du lot A_simplif. En effet, une faible prise alimentaire a été observée chez les animaux du Tém_E et ceux du lot A_simplif. Ces résultats, à eux seuls, expliquent les faibles performances pondérales des animaux. Comme indiqué précédemment, la présence de substances indésirables dans le tourteau ou l'huile d'arachide peut rendre compte de ces faibles consommations dans le lot témoin ; chez ceux recevant la ration simplifiée, le faible nombre d'aliments composant le mélange pourrait expliquer cette faible ingestion.

Les croissances plus importantes observées chez les animaux recevant la fève de coton glandless par rapport à celles du témoin expérimental ont été également liées à une ingestion alimentaire plus importantes $(+13,63$ et $+11,55 \mathrm{~g}$, respectivement au démarrage et en croissance). Ces plus fortes ingestions sont vraisemblablement expliquées par une appétence élevée des provendes liée à la fève de coton glandless. Ce phénomène avait déjà été observé par Diaw et coll. (7). Il semble donc que l'amande de coton exempte de gossypol stimule l'ingestion chez les animaux (16). Des résultats similaires ont été obtenus, par plusieurs auteurs, après détoxification du tourteau de coton $(13,16,31)$. Toutefois, cette hypothèse n'a pas été vérifiée dans le cas de la ration simplifiée.

Les indices de consommation obtenus (1,53 à 2,34 en démarrage et 1,89 à 3,54 en croissance) ont été supérieurs à ceux rapportés par certains auteurs $(13,31)($ de 1,43 à 1,59 , et de 1,41 à 1,94 , respectivement en démarrage et en croissance), mais de telles perforN mances sont extrêmes. Après 63 jours d'élevage, Ojewola et Ewa (22) ont obtenu des IC de 2,31 à 2,51. Chez le Cobb 500, des IC de 1,49 et 1,99 ont été obtenus en station avec respectivement des aliments démarrage et croissance, mais dans des conditions idéales de température et d'humidité (29).

Il faut souligner la très bonne efficience alimentaire de la ration simplifiée durant la phase de croissance $(1,89)$, appuyant son adoption en aviculture traditionnelle où la vitesse de croissance n'est pas nécessairement une priorité. La question de faible performance dépend donc du contexte dans lequel on l'étudie et, par rapport à l'objet même de cette ration simplifiée qui vise les éleveurs amateurs, elle devrait être intégrée dans la dimension socio-économique de l'aviculture traditionnelle. En effet, avec ce type d'élevage caractérisé surtout par la divagation, les animaux réalisent des croîts très faibles qui, au-delà de leurs potentiels génétiques, seraient liés à une mauvaise valorisation des ressources locales.

Les mortalités enregistrées au démarrage dépassent la norme en vigueur au Sénégal qui est de 5 p. 100 (3). Elles sont vraisemblablement liées à l'ambiance climatique. En revanche en croissance, l'analyse de survie situe les mortalités principalement au niveau du lot d'animaux recevant la ration simplifiée. Ce problème remet au premier plan le caractère sans doute trop simplifié de cet aliment qui, en entraînant une trop faible consommation, a probablement affaibli les animaux élevés en claustration totale, sans qu'il soit toutefois possible d'identifier la cause première du phénomène (animaux affaiblis parce que mangeant peu ou inversement). Toutefois, en conditions traditionnelles, ce problème devrait être évité, les animaux diversifiant naturellement leur ration.

\section{CONCLUSION}

Les résultats obtenus montrent que la fève de coton glandless constitue un excellent aliment en production de poulets. Son utilisation offre, au Sénégal, la possibilité de couvrir le déficit de la demande des fabriques d'aliment en tourteau d'arachide consécutif à la crise de la filière arachidière et au développement de l'aviculture, ainsi qu'une amélioration de la nutrition en aviculture traditionnelle qui contribue au développement socio-économique en milieu rural, en particulier dans la zone cotonnière. La plus-value engendrée sur les productions du cotonnier pourrait ainsi améliorer la rentabilité de la culture auprès des producteurs.

\section{Remerciements}

Ce travail a été effectué dans le cadre du « Projet interuniversitaire ciblé » portant sur l'amélioration de la rentabilité de la filière cotonnière sénégalaise. Les auteurs remercient la Commission universitaire au développement de la Belgique pour avoir financé cette étude.

\section{BIBLIOGRAPHIE}

1. AOAC, 1990. Official methods of analysis, 15th Edn. Washington, DC, USA, Association of Official Analytical Chemists.

2. AZMAN M.A., YILMAZ M., 2005. The growth performance of broiler chicks fed with diets containing CSM supplemented with lysine. Rev. Méd. vét., 156: 104-106.

3. BULDGEN A., 1996. Aviculture semi-industrielle en climat subtropical. Gembloux, Belgique, Presses agronomiques, $122 \mathrm{p}$.

4. CZEGLEDI L., GUTZWILLER A., 2006. Mycotoxines dans les céréales et les aliments pour les animaux en Suisse : revue de la littérature. Rev. Suisse Agric., 38 : 329-340.

5. DAHOUDA M., TOLEBA S.S., YOUSSAO A.K.I., HAMBUCKERS A., DANGOU-SAPOHO R., MARTIN G.B., FILLET M., HORNICK J.L., 2009. Nutrient digestibility of Mucuna (Mucuna pruriens var. utilis) bean in guinea fowl (Numida meleagris, L): Effects of heat treatment and levels of incorporation in diets. Br. Poult. Sci., 50: 564-572.

6. DIAW M.T., DIENG A., MERGEAI G., SY M., HORNICK J.L., 2010. Effets de la substitution du tourteau d'arachide par la fève de coton conventionnel en production de poulets de chair au Sénégal. Tropicultura, 28 : 139-147.

7. DIAW M.T., DIENG A., MERGEAI G., YOUSSOUF I., DOTTREPPE O., HORNICK J.L., 2010. Effect of groundnut cake substitution b glandless cottonseed kernels on broilers production: Animal performance, nutrient digestibility, carcass characteristics and fatty acid composition of muscle and fat. Int. J. Poult. Sci., 9: 473-481.

8. DIAW M.T., DIENG A., MERGEAI G., HORNICK J.L., 2011. Les coproduits de la graine de coton en alimentation du poulet de chair. Ann. Méd. vét., 155 : 61-82.

9. FERNANDEZ S.R., ZHANG Y., PARSON C.M., 1995. Dietary formulation with cottonseed meal on a total amino-acid versus a digestible amino acid basis. Poult. Sci., 74: 1168-1179.

10. FOSTA J.-C., MANJELI Y., 2001. Analyse comparée des performances de croissance en claustration des poussins de souche locale, d'une lignée Jupiter, et de leurs croissements F1. Ann. Sci. Agron. Bénin, 2 : 181-192.

11. GAMBOA D.A., CALHOUN M.C., KUHLMANN S.W., HAQ A.U., BAILEY C.A., 2001. Tissue distribution of gossypol enantiomers in broilers fed various cottonseed meals. Poult. Sci., 80: 920-925.

12. GUEYE E.F., 2000. The role of family poultry in poverty alleviation, food security and the promotion of gender equality in rural Africa. Outlook Agric, 29: 129-136.

13. HENRY M.H., PESTI G.M., BAKALLI R., LEE J., TOLEDO R.T., EITENMILLER R.R., PHILLIPS R.D., 2001. The performance of broiler chicks fed diets containing extruded cottonseed meal with lysine. Poult. SCi., 80: 762-768.

14. HRON R.J., WAN P.J., KEEK M.S., 1994. Ethanol vapor deactivation of gossypol in cottonseed meal. J. Am. Oil Chem. Soc., 73: 1337-1339. 
15. INSTITUT NATIONAL RECHERCHE AGRONOMIQUE, 1987. Nutrition of laying hens. In: Wiseman J. Ed., Feeding of non-ruminant livestock. London, UK, Butterworths, $214 \mathrm{p}$.

16. KETEKOU C.S., 1985. Intérêt biologique de l'huile de graine de coton. In : Le cotonnier sans gossypol, une nouvelle ressource alimentaire. Abidjan, Côte-d'Ivoire, Idessa, p. 58-62.

17. MALCOLM REID W., PESTI G.M., HARGIS B., MOORE R., VOHRA P., DEAN W.F., HAMMARLUND M.A., 2006. Raising healthy poultry. Seattle, WA, USA, Christian Veterinary Mission, $138 \mathrm{p}$.

18. MERGEAI G., 2003. Forty years of genetic improvement of cotton through interspecific hybridisation at Gembloux Agriculture University: Achievement and prospects. In: $3^{\text {rd }}$ World Cotton Research Conference, Cape Town, South Africa, 9-13 March 2003.

19. NAGALAKSHMI D., SAVARAN V., RAMA R., ARUN K.P., VADALI R.B.S., 2007. Cottonseed meal in poultry diets: A review. J. Poult. Sci., 44: 119-134.

20. NATIONAL RESEARCH COUNCIL, 1994. Nutrient requirements of poultry, $9^{\text {th }}$ Edn. Washington, DC, USA, National Academy Press.

21. NDEGWA I.M., MEAD R., NORRISH P., KIMANI C.W., WACHIRA A.M., 2001. Performance of indigenous Kenyan chickens fed diets containing different levels of protein during rearing. Trop. Anim. Health Prod., 33: 441-448.

22. OJEWOLA G.S., EWA U.E., 2005. Response of growing broiler to varying dietary plant protein. J. Poult. Sci., 4: 765-771.

23. PANIGRAHI S., 1992. Effects of treating cottonseed meal with a solution of ferrous sulphate on laying hen performance and discolouration in eggs. Anim. Feed Sci. Technol., 38: 89-103.

24. PANIGRAHI S., PLUMB V.E., 1996. Effects of dietary phosphorus of treating cottonseed meal with crystalline ferrous sulphate for the prevention of brown yolk discolouration. Poult. Sci., 37: 403-411.

25. SAS, 2000. Statistics users guide, Statistical analysis system, 5th Edn, Vers. 8.2. Carry, NC, USA, SAS Institute.

\section{Summary}

Diaw M.T., Dieng A., Mergeai G., Camara A., Hornick J.L. Effect of the total substitution of glandless cottonseed kernels for groundnut cake on broiler production in Senegal

The performances of broilers fed a diet in which the groundnut cake was totally replaced by glandless cottonseed kernels (FCG) were compared to those of animals fed an experimental control diet (Tém_E), a commercial diet (Tém_C), and a very simple diet containing only corn, FCG and a mineral and vitamin concentrate. From the start, the Tém_C and FCG animals showed the highest growths. After 43 days of breeding, there were major differences in body weights between the groups, essentially caused by differences in feed intake, so that the best growth rates were not necessarily linked to better feed conversion ratios. Low body weights, low intakes and high mortality levels were observed with the simple diet.

Keywords: Poultry - Broiler chicken - Glandless cotton Feeding - Animal performance - Senegal.
26. REDDY R., ESHWARAIAH V., 1987. Studies on the utilization of decorticated deoiled, double-toasted cottonseed extraction broiler starters. Indian J. Poult. Sci., 22: 194-196.

27. SCHMIDELY P., SAUVANT D., 2001. Taux butyreux et composition de la matière grasse du lait chez les petits ruminants : effets de l'apport de matière grasse ou d'aliment concentré. Prod. Anim., 14 : 337-354.

28. SHARMA N.K., LODHI G.N., ICHHPONANI J.S., 1978. Comparative feeding value of expeller processed undecorticated and decorticated cottonseed cakes for growing chicks. J. Agric. Sci., 91: 531-541.

29. TEETER R., WIERNUSZ C., 2003. Cobb broiler nutrition guide. http:// www.cobb-vantress.com/contactus/brochures/broilernutritionguide.pdf (consulté le 22.02.2007).

30. TRAORE E.H., 2006. Première évaluation de la structure et de I'importance du secteur avicole commercial et familial en Afrique de I'Ouest : Rapport du Sénégal. Rome, Italie, FAO, 52 p.

31. WATKINS S.E., SKINNER J.T., ADAMS M.H., WALDROUP P.W., 1993. An evaluation of low-gossypol cottonseed meal in diets for broiler chickens: Effect of cottonseed meal level and lysine supplementation. I. Appl. Poult. Res., 2: 221-226.

32. WEN-JU Z., ZI-RONG X., JIAN-YI S., XIA Y., 2006. Effect of selected fungi on the reduction of gossypol levels and nutritional value during solid substrate fermentation of cottonseed meal. J. Zhejiang Univ. Sci., 7: 690-695.

33. YO T., 1991. Utilisation directe de graines de cotons décortiquées de variétés sans gossypol dans I'alimentation des poulets de chair en Côte d'Ivoire. Rev. Elev. Méd. vét. Pays trop., 44 : 355-360.

34. YU F., BARRY T.N., MOUGHAN P.J., WILSON G.F., 1993. Condensed tanin and gossypol concentrations in cottonseed and in processed cottonseed meal. J. Sci. Food Agric., 63: 7-15.

Accepté le 11.09.2012

\section{Resumen}

Diaw M.T., Dieng A., Mergeai G., Camara A., Hornick J.L. Efectos de la sustitución total de torta de cacahuete por semilla de algodón sin glándulas en el rendimiento de pollos de engorde en Senegal

El rendimiento de los pollos de engorde alimentados con un alimento en el cual la torta de cacahuete ha sido totalmente sustituida por semilla de algodón sin glándulas, se comparó con el de los animales alimentados con una dieta testigo experimental (Test._E), una dieta comercial (Test._C) y una ración de formulación muy simple preparada solamente con maíz, semillas de algodón sin glándulas y concentrado con vitaminas y minerales. Desde el comienzo, los animales Test._C y semillas de algodón sin glándulas revelaron los mejores crecimientos. A los 43 días de cría, las diferencias en los pesos corporales eran muy importantes entre los grupos, debido principalmente a las diferencias en el consumo de alimentos, de tal manera que las mejores tasas de crecimiento no se relacionaron necesariamente con los mejores índices de consumo. Fueron observados bajos crecimientos ponderales, bajos consumos e importantes tasas de mortalidad con la dieta simplificada.

Palabras clave: Ave de corral - Pollo de engorde - Algodón sin glándulas - Alimentación - Desempeño animal - Senegal. 
